\title{
Reabilitação estética e funcional em paciente com amelogênese imperfeita: relato de caso
}

\author{
Aesthetic and functional rehabilitation in a patient \\ with imperfect amelogenesis: a case report
}

\author{
Rafaela Costa Holanda' \\ Diana Maria Arraes Feitosa' \\ Ginna Kércia Matos Gonçalves' \\ Karla Shangela Silva Alves" \\ Thayla Hellen Nunes Gouveia \\ Patrícia Leal Dantas Lobo IV \\ 'Academia Cearense de Odontologia (ACO). Fortaleza/CE - Brasil \\ "Centro Universitário Católica de Quixadá (UNICATÓLICA). Quixadá/CE - Brasil \\ "'Universidade Estadual de Campinas (UNICAMP). Piracicaba/SP - Brasil \\ IVUniversidade Federal do Ceará (UFC). Sobral/CE - Brasil
}

\begin{abstract}
RESUMO
Introdução: A amelogênese Imperfeita constitui um distúrbio de caráter hereditário caracterizado pela formação anormal do esmalte, podendo ocorrer tanto na dentição decídua, como na permanente. O esmalte pode apresentar-se como hipoplásico, hipomaturado, hipocalcificado ou hipoplásico-hipomaturado e com taurodontismo, surgindo de forma isolada ou associada a outras anomalias. Sua etiologia pode ser de origem local, sistêmica, genética ou idiopática. O diagnóstico deve ser preciso para proceder ao tratamento adequado para cada caso, seja preventivo, interceptativo ou acompanhamento.

Objetivo: Este artigo tem como objetivo apresentar um caso clínico de um paciente com por amelogênese imperfeita, o qual foi reabilitado estética e funcionalmente, por meio da técnica direta, utilizando resinas compostas.

Relato de caso: O relato deste caso refere-se a uma paciente do gênero feminino, 11 anos de idade, que apresentou queixa de "dentes escuros", "vergonha de sorrir" e "dificuldade de higienizar", representando sério comprometimento estético e funcional. Foi realizado incluindo fase preventiva, restauradora e de manutenção, devolvendo estética e função à paciente.

Conclusão: O tratamento proposto mostrou ser eficaz, minimizando desde problemas estéticos e funcionais, até interferir em seu convívio social e suas relações pessoais, devolvendo à paciente uma estética favorável e ausência de sensibilidade nos dentes.
\end{abstract}

Palavras-chaves: Amelogênese imperfeita. Estética dental. Reabilitação oral.

\section{ABSTRACT}

Introduction: The Imperfect Amelogenesis constitutes a hereditary disorder characterized by the abnormal formation of enamel, which may occur in the primary dentition as well as in the permanent dentition. The enamel may present as hypoplastic, hypomaturated, hypocalcified or hypoplastic-hypomaturated with taurodontism, appearing in isolation or associated with other anomalies. Its etiology may be from local, systemic, genetic or idiopathic origin. The diagnosis must be precise in order to proceed with the appropriate treatment for each case, be it preventive, interceptive or clinical follow-up.

Aim: This article aims to present a clinical case of a patient with imperfect amelogenesis, which was aesthetically and functionally rehabilitated by means of the direct restorative technique using composite resins.

Case report: The report of the present case refers to a female patient, 11 years old, who presented a complaint of "dark teeth", "shame of smiling" and "difficult on oral hygiene", presenting a serious aesthetic and functional impairment. This case was carried out including the preventive, restorative and maintenance phases, returning aesthetics and function to the patient.

Conclusion: The proposed treatment proved to be effective, minimizing from aesthetic and functional problems to interfere with social interaction and personal relationships, giving the patient a favorable aesthetic and lack of sensitivity in the teeth.

Key-words: Imperfect Amelogenesis. Dental Aesthetics. Oral rehabilitation. 


\section{INTRODUÇÃO}

A busca pelo sorriso estético e harmônico tem ocupado grande espaço e destaque na odontologia, levando a inúmeros avanços nessa área, proporcionando cada vez mais possibilidades e alternativas que objetivam reparar ou corrigir a aparência dos dentes comprometidos, como é o caso da Amelogênese Imperfeita (Al).

A presença dessa alteração dentária causa um impacto psicológico ao paciente, além de problemas de socialização, função e desconforto, mas pode ser contornada com tratamento continuado durante toda a infância e até a vida adulta. ${ }^{1}$

A Al se refere a um grupo de doenças hereditárias de formação do esmalte, com manifestação similar nas duas dentições, tanto na decídua quanto na permanente. ${ }^{2} \mathrm{~A}$ causa desses defeitos, na formação ou na mineralização do esmalte, durante a amelogênese, pode ser geralmente classificada como sistêmica, genética ou local. ${ }^{3} \mathrm{O}$ fator hereditário é provavelmente um distúrbio generalizado dos ameloblastos e os fatores locais afetam dentes isolados e, em muitos casos, um único dente. ${ }^{4,5}$

A classificação da Al é complexa. A mais amplamente utilizada foi a proposta por Witkop,$^{6}$ que a dividiu em quatro tipos: tipo I - hipoplásica - o defeito ocorre na formação da matriz do esmalte, que se mostra fino e com presença de sulcos e fossas; tipo II - hipomaturada - o defeito ocorre na maturação do esmalte, exibindo espessura e dureza normais, mas com um manchamento opaco, variando de branco a amarelo-amarronzado, ou vermeIho-amarronzado; tipo III - hipocalcificada - o defeito ocorre na mineralização do esmalte e, portanto, este se mostra áspero, descolorido e com menor consistência; tipo IV - hipomaturada - hipoplásica é uma combinação das duas com presença de taurodontismo. Assim, as formas da Al variam desde a presença de um esmalte deficiente, até a ocorrência de defeitos no conteúdo mineral e proteico ${ }^{5}$ e podem ser identificadas com base nas características clínicas, radiográficas, histológicas e composição do esmalte dos dentes. ${ }^{7}$

As manifestações clínicas encontrados na Al são variáveis, podendo desencadear impulso lingual, levando à mordida aberta anterior e aos defeitos estruturais que provocam a diminuição da dimensão vertical, perda extensiva de tecido dental, comprometimento da estética e da sensibilidade dentária. Com o aumento de retenção de placa dental, devido à superfície rugosa do esmalte e à dificuldade de higienização, a inflamação gengival também está relacionada a Al. ${ }^{1}$

Desse modo, o diagnóstico da Al é realizado a partir das características clínicas, radiográficas e no modo da herança, onde deve ser feito corretamente e o mais precoce possível, contribuindo para minimizar as sequelas provenientes dessa anomalia, bem como, executar o planejamento que se enquadre melhor para cada caso. ${ }^{8}$

O tratamento é ancorado no tipo de gravidade da desordem e de outros fatores, como a idade do paciente, a condição socioeconômica e o estado de saúde bucal, no momento da elaboração do planejamento. ${ }^{9,10}$

Podemos mencionar, como formas de tratamento, as extrações dentárias, restaurações estéticas conservadoras, confecção de coroas, controle da sensibilidade dentinária e orientação de higiene bucal, sendo a abordagem multidisciplinar um fator fundamental para o sucesso do tratamento. ${ }^{4,11}$ Atualmente, as restaurações de resinas compostas podem ser indicadas e colocadas com mínima ou nenhuma preparação dentária, para preservar a estrutura e proporcionar estética satisfatória. ${ }^{12}$

O objetivo do presente estudo foi descrever um caso clínico de Al, reabilitado a partir de restaurações adesivas diretas, devolven- 
do função e estética ao paciente que, de um modo geral, interferirá de forma positiva na sua qualidade de vida.

\section{Caso clínico}

O artigo foi submetido e aprovado pelo Comitê de Ética em Pesquisa do Centro de Educação Continuada da Academia Cearense de Odontologia com o número 1.356.977. A publicação deste estudo foi autorizada pelo responsável da criança por meio da assinatura de um termo de consentimento livre e esclarecido.

Paciente do sexo feminino, 11 anos de idade, apresentou-se à Clínica de Especialização em Odontopediatria do Centro de Educação Continuada Perboyre Castelo, da Academia Cearense de Odontologia, acompanhada da sua responsável, queixando-se do aspecto amarelado dos seus dentes, do desconforto ao comer, da sensibilidade dental ao ingerir comidas quentes e geladas e de não conseguir escovar os dentes devido à sensibilidade dentária no momento da higienização. Durante a anamnese, a mãe relatou que a paciente apresentava dentes de leite com esse mesmo tipo de má-formação. Quando questionada a respeito dessa anomalia em algum membro da família, a mãe disse que ninguém da família tinha esse tipo de problema nos dentes. No exame clínico, observaram-se manchas castanhas-amareladas em todos os dentes, exceto os dentes 11, 12, 21 e 22, que mostravam restaurações insatisfatórias; os outros dentes encontravam-se com desgaste dental generalizado, coroa curta, cárie aguda no elemento 31, higiene dental deficiente, presença de cálculo dental, gengivite generalizada e mordida aberta anterior (Figura 1).

O quadro clínico e radiográfico revelou a ausência de esmalte dental em alguns locais e uma fina camada do tecido em outros, dentina e cavidade pulpar com aspecto de normalidade e raízes com ausência de alteração (Figura 2).

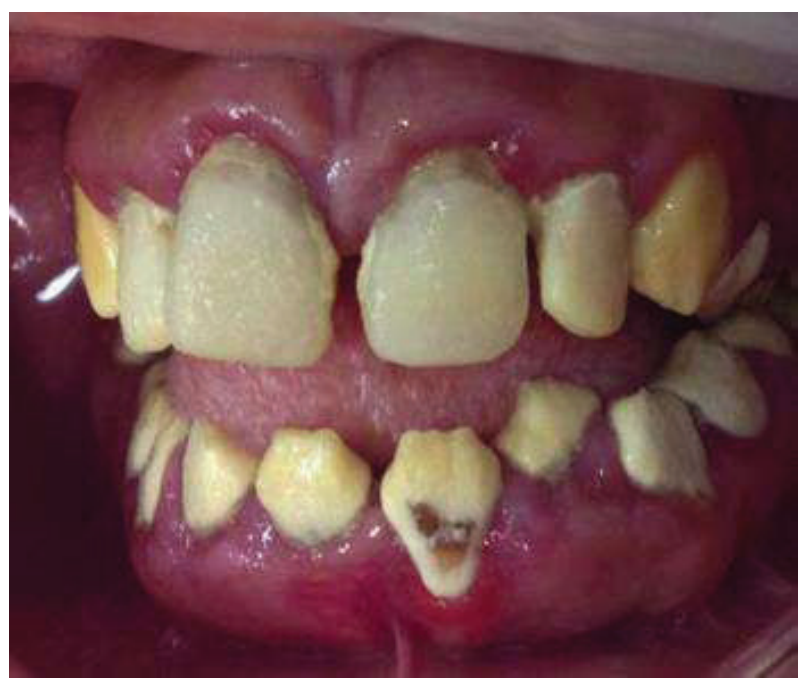

Figura 1: Aspecto inicial.

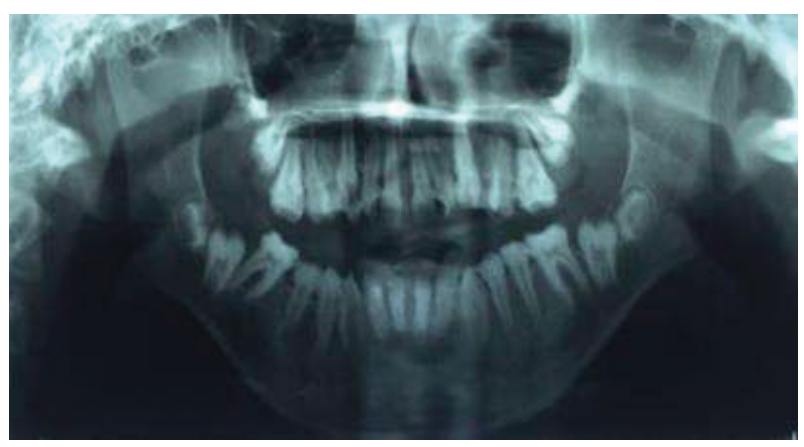

Figura 2: Radiografia panorâmica.

Para a realização do plano de tratamento, levaram-se em consideração os desejos e expectativas do paciente, nível socioeconômico, idade do paciente e severidade da Al. Optou-se por um tratamento conservador, composto por restaurações diretas com resina composta, considerando a importância do tratamento, não apenas do ponto de vista funcional e estético, como também, sua influência na qualidade de vida do paciente.

O tratamento foi realizado em 24 sessões, incluindo as fases preventiva, restauradora e de manutenção, com o objetivo de devolver estética e função ao paciente, facilitar a higiene e promover uma melhor saúde bucal. 
Iniciou-se pela terapia periodontal, por meio da profilaxia, raspagem supragengival seguida por uma gengivoplastia, inicialmente, na região mandibular (Figura 3) e, posteriormente, na região maxilar, a fim de facilitar a manutenção da saúde periodontal e melhor adaptação das restaurações. Em todas as sessões foi aplicado o adesivo universal, para reduzir a sensibilidade dental e facilitar a higiene bucal, em função do alto grau de sensibilidade, até que todos os dentes fossem restaurados.

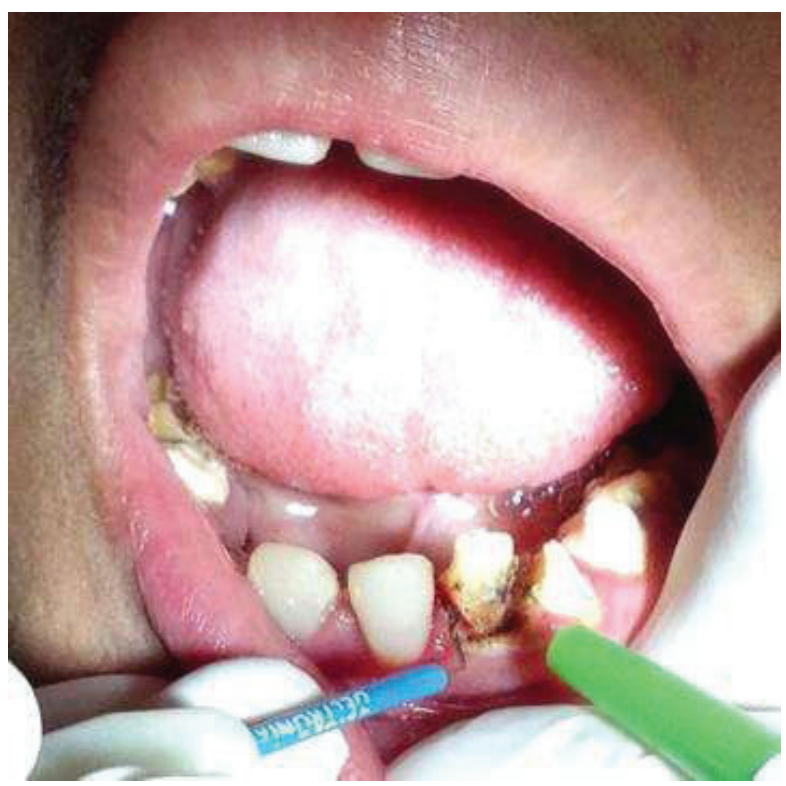

Figura 3: Gengivoplastia.

Seguindo o planejamento, o tratamento foi realizado onde os elementos $11,12,13$, $14,15,21,22,23,24,25,31,32,33,34,35$, $41,42,43,44$, e 45 foram restaurados, tendo como material de escolha a resina composta (Figura 4-8).

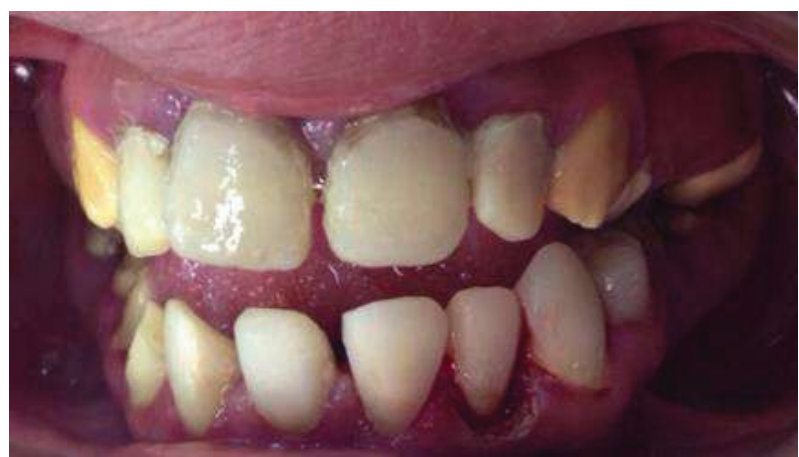

Figura 4: RestauRações dos ElEMENTOS DENTÁRIOS (33 e 34).

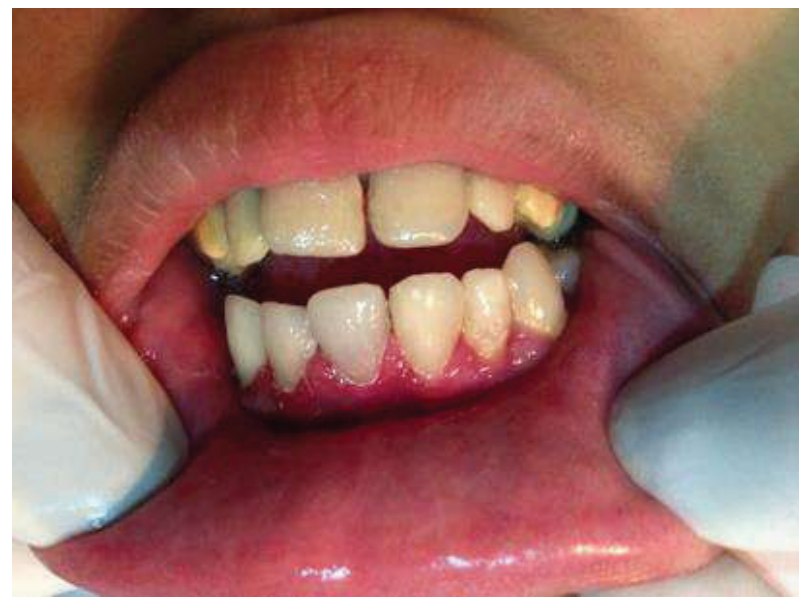

FIGURA 5: RESTAURAÇõES dOS ELEMENTOS DENTÁRIOS (31, 32, 41 E 42).

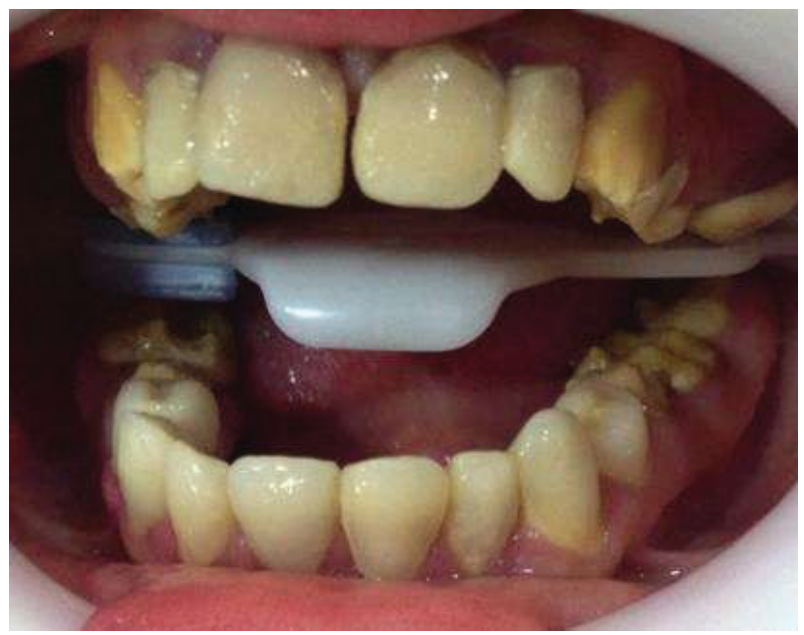

Figura 6: RestauRAÇõES dos ELEMENTOS DENTÁRIOS (34 E 44).

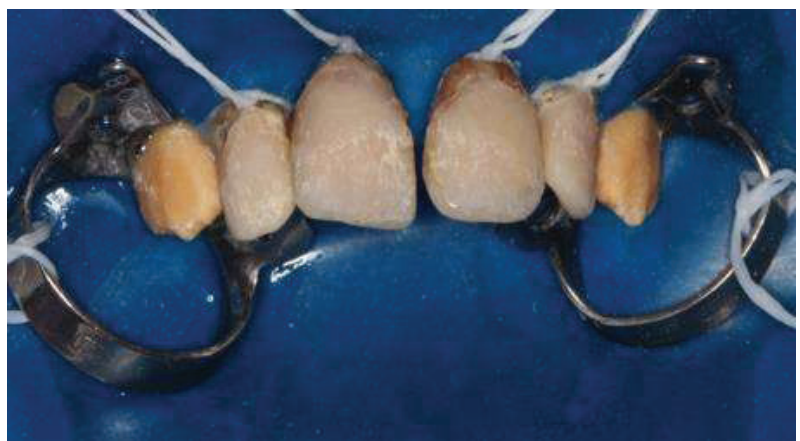

FIgURA 7: RESTAURAÇÕES INSATISFATÓRIAS NOS INCISIVOS SUPERIORES $(11,12,21$, E 22). 


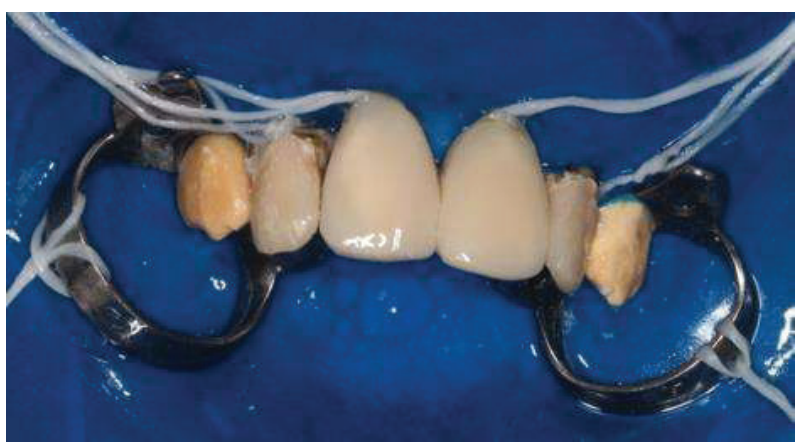

Figura 8: Restaurações nos incisivos centrais superiores (11 e 21).

Após a cirurgia periodontal e a realização das restaurações com resina composta, que promoveram a reconstrução dos dentes, foi possível adequar o alinhamento tanto do arco superior, quanto do inferior, e uma diminuição considerável da mordida aberta anterior, quando comparada ao aspecto inicial da paciente. $\mathrm{Na}$ execução do planejamento, houve essa preocupação de promover o alinhamento dos arcos dentários e diminuição da mordida aberta anterior, comprovadas nas figuras relacionadas (Figura 9 e 10).

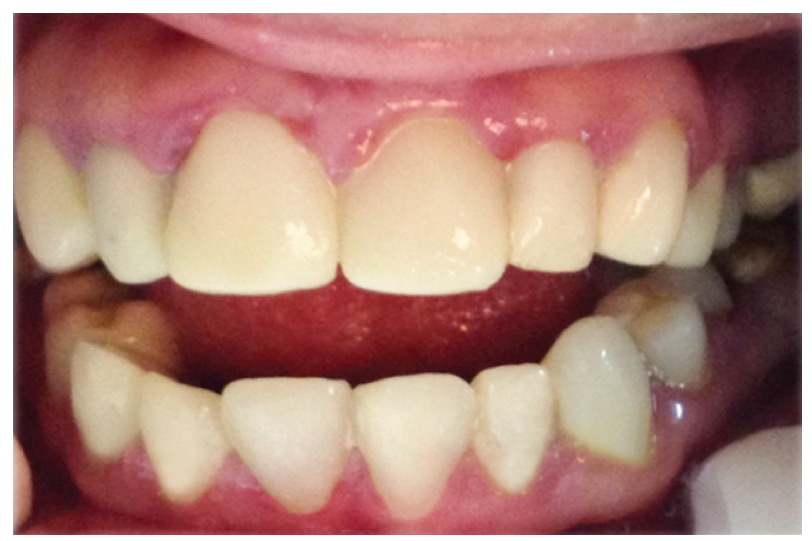

Figura 9: Aspecto final do tratamento.

Ao final do tratamento, a paciente relatou as melhoras significativas que ocorreram. Mencionou que o tratamento colaborou diretamente na elevação de sua autoestima, isto decorrente da melhoria do aspecto do seu sorriso, possibilitando-lhe uma melhor interação

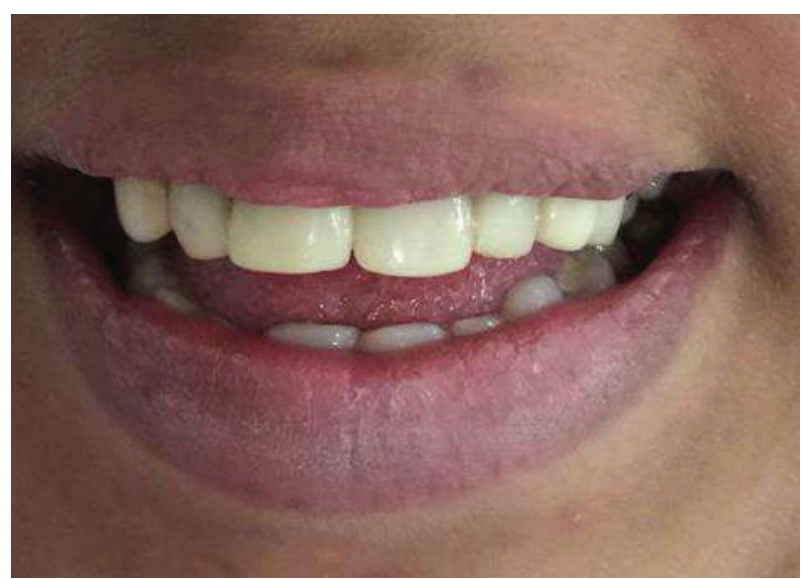

FIgURA 10: AsPeCto FINAL DO SORRISO DA PACIENTE, APÓS O TRATAMENTO.

social. Salienta que, após o término do tratamento, conseguia escovar os dentes e comer sem ter sensibilidade, fato que, anteriormente ao tratamento, não era possível. Dessa forma, percebe-se o quanto esse tratamento interferiu na sua qualidade de vida e impactou nos aspectos estéticos e funcionais.

Foram feitas recomendações quanto à higienização e dieta para a preservação das resinas e manutenção da saúde oral de forma adequada, com visitas regulares ao dentista e quanto à necessidade da continuidade do tratamento, como a reabilitação dos molares.

\section{Dıscussão}

É inquestionável que um sorriso harmônico tem sido valorizado pela sociedade atual, apresentando influência direta relacionada à autoestima e convívio social. Este trabalho correspondeu às expectativas da paciente, recuperando e promovendo um sorriso harmônico e saudável com a diminuição da sensibilidade dental, possibilitando uma adequada higienização e realizando uma melhora clínica significativa no aspecto funcional e estético do sorriso. Esse relato mostrou grande impacto em sua vida, afetando positivamente a autoestima e o convívio social. Oliveira et al. ${ }^{13}$ relatam que essa baixa autoestima pode 
diminuir o desempenho escolar e favorecer o isolamento social.

Com base na anamnese, queixa do paciente, exame clínico e radiográfico e na ausência de alterações sistêmicas, confirmou-se o diagnóstico de Al do tipo hipocalcificada no caso apresentado. A cor fosca exibida pelo esmalte, nesse caso, representa baixa mineralização, clinicamente expressa pela estrutura do esmalte pigmentado, suavizado e facilmente destacável. Essas características auxiliaram no direcionamento do diagnóstico, características também relatadas por Marquezin et al. ${ }^{14}$

Além dos sinais já descritos, observa-se a presença de dentes com alteração de forma, tamanho e coloração, recobertos por uma fina camada de esmalte, apresentando-se rugoso e ausente em algumas áreas. Também constatou-se quadro de gengivite generalizada, em razão do acúmulo de placa bacteriana provocado pelas irregularidades do esmalte e pela dificuldade de higienização, devido à sensibilidade dental apresentada, conforme relatado em alguns trabalhos. ${ }^{15,16}$

Com relação aos achados clínicos, sabe-se que pacientes com Al apresentam problemas primários como: lesões de cárie, inflamação gengival, estética comprometida e sensibilidade dental, ${ }^{17}$ todos identificados no caso.

Apesar de a mãe não ter relatado a presença de alteração em outros membros da família, questiona-se a capacidade da mãe da paciente de identificar casos mais suaves da anomalia em outros membros da família. ${ }^{5}$

Segundo estudo realizado por Nathwani NS e Kelleher $\mathrm{M},{ }^{18}$ a Al provoca hipersensibilidade dentária, ausência de pontos de contato, perda de função e de estética, fatos que justificam intervenção o mais cedo possível, a fim de evitar desenvolvimento de cárie, doença periodontal e perda de dimensão vertical. No caso abordado, foi relatado que, após a erupção dos dentes permanentes, a paciente procurou atendimento odontológico e iniciou o tratamento restaurador, onde não foi possível concluir o tratamento no primeiro momento. Após algum tempo, em outra oportunidade, ela, novamente, buscou atendimento para realizar o tratamento, relatando da insatisfação das restaurações realizadas, dificuldade ao comer, ingerir alimentos em altas ou baixas temperaturas e impossibilidade de higienizar os dentes decorrentes da sensibilidade dental. Diante do exposto, o tratamento foi planejado e executado conforme já relatado.

Independentemente da técnica, ou material escolhido, é importante que o plano de tratamento abranja todas as seguintes fases: preventiva (incluindo instrução de higiene bucal e redução da sensibilidade dental), restauradora e de manutenção. Os profissionais também devem estar atentos acerca da importância das avaliações de retorno, para monitorar a higiene e a condição periodontal e pulpar, como parte do tratamento. ${ }^{19,20}$

O tratamento para essa anomalia é muito variável, porque depende do tipo e gravidade da desordem para se realizar um trabalho adequado. Saber diagnosticar e classificar corretamente essa anomalia, numa abordagem multidisciplinar, é fator fundamental para um tratamento de sucesso. ${ }^{9}$

No caso de pacientes jovens, a finalidade do tratamento é manter a máxima quantidade de estrutura dentária possível até que estes alcancem uma idade em que possam ser utilizadas técnicas restauradoras avançadas para a reabilitação dental. $\mathrm{O}$ aspecto clínico do esmalte durante a preparação do dente desempenha um papel decisivo. Em alguns casos de $\mathrm{Al}$, é comum a necessidade de alongamento coronário, ${ }^{8}$ como descrito neste caso.

Por muitos anos, a mais previsível e durável restauração estética de dentes anteriores era alcançada apenas com coroas completas. ${ }^{21}$ Contudo, esse tratamento requer a remoção de grande quantidade de estrutura dentária sadia e representa um alto custo para 
o paciente. Esse caso ilustra que, utilizando somente restaurações diretas, é possível reabilitar um paciente com amelogênese imperfeita, indo de encontro ao estudo de Figueiredo et al., ${ }^{1}$ demonstrando que, usando técnicas conservadoras e de baixo custo, restaurações com resinas compostas podem alcançar a estética desejada e ter as estruturas de suportes preservadas.

O material de escolha para a realização deste caso foi a resina composta direta, seguindo o tratamento proposto por Felippe et al., ${ }^{22}$ que destacam que técnicas conservadoras devem ser as primeiras opções. Nesse sentido, o aprimoramento das resinas compostas aumenta sua indicação, inclusive para dentes posteriores.

Este trabalho apresenta uma alternativa terapêutica conservadora para a promoção e recuperação da estética e função em uma paciente com amelogênese imperfeita, buscando uma melhor qualidade de vida.

\section{Considerações finaIS}

A Al pode apresentar quadros complexos, sendo o diagnóstico correto e precoce e o tratamento restaurador e preventivo essenciais para o sucesso do tratamento dessa anomalia. O cuidadoso planejamento associado a um adequado acompanhamento permitirá que o paciente alcance uma dentição com características estético-funcionais satisfatórias.

A situação clínica relatada mostra que os avanços no desenvolvimento dos materiais adesivos, aliados às técnicas de preparo minimamente invasivas, permitem a resolução de situações clínicas com eficiência estética e funcional, como neste caso de Al.

\section{REFERÊNCIAS}

1. Figueiredo RG, Moreira RF, Simões OS, Novaes SEA, Fonseca ACL, Miranda MS. Amelogênese imperfeita: A importância do diagnóstico precoce na saúde e no desenvolvimento do adolescente. Relato de caso. Rev Adolesc e Saúde, 2016; 13 (2): 87-93.

2. Leevailoj C., Lawanrattanakul S., Mahatumarat K. Amelogenesis Imperfecta: Case Study. Oper Dent., 2017; 42 (5): 457-469.

3. Smith RN, Elcock C., Abdellatif A., Bäckman B., Russell JM, Brook AH. Enamel defects in extracted and exfoliated teeth from patients with Amelogenesis Imperfecta, measured using the extended enamel defects index and image analysis. Arch Oral Biol., 2009; 5 (1): 86-92.

4. Kammoun R., Behets C., Mansour L., GhoulMazgar S. Mineral features of connective dental hard tissues in hypoplastic amelogenesis imperfecta. Oral Dis., 2017, Aug 3.

5. Gonçalves, AF, Ferreira, SLM. Defeitos hipoplásicos do esmalte dentário. $\mathbf{R}$ Odont Univ., 2000; 5 (1): 13-20.

6. Witkop JR. DJ. Amelogenesis imperfecta, dentinogenesis imperfecta and dentin dysplasia revisited: problems in classification. J Oral Pathol., 1989; 17 (9-10): 547-553.

7. Sarbeen JI, Ranjan M. Functional and esthetic rehabillitation of patients with amelogenesis imperfect. 2017; An International Open Access Journal., 2017; 2 (4): 157-160.

8. Brizola E., Zambranob MB, Pinheiro BS, Vanza AP, Félixa TM. Clinical features and pattern of fractures at the time of diagnosis of osteogenesis imperfecta in children. Rev Paul Pediatr., 2017; 35 (2): 171-177.

9. Azevedo MS, Goettems ML, Torriani DD, Romano AR, Demarco FF. Amelogenesis imperfecta: clinical aspects and treatment. RGO., 2013; 61: 491-496.

10. McDonald S., Arkutu N., Malik K., Gadhia K., McKaig S. Managing the pediatric patient with amelogenesis imperfecta. British Dental Journal., 2012; 212 (9): 425-428.

11. Turkun LS, Turkey I. Conservative restoration with resin composites of a case of amelogenesis imperfecta. Int Dent Journal., 2005; 55: 38-41.

12. Rogers HJ, Yesudian G., Rodd HD. Unusual extrinsic staining following microabrasion in a girl with amelogenesis imperfecta. Eur Arch Paediatr Dent. 2016; 17(4): 271-5.

13. Oliveira AF, Chaves AM, Rosenblatt A. The influence of enamel defects on the development 
of early childhood caries in a population with low socioeconomic status: a longitudinal study. Caries Res., 2006; 40(4): 296-302.

14. Marquezin MC, Zancopé BR, Pacheco LF, Gavião MB, Pascon FM. Aesthetic and functional rehabilitation of the primary dentition affected by amelogenesis imperfecta. Case Rep Dent., 2015; 2015: 790-890.

15. Santos MCLG, Line SRP. The genetic of amelogenesis imperfecta. A review of the literature. J Appl Oral Sci., 2005; 13 (3): 212217.

16. Encinas RP, Espona IG, Módelo JMNRDE. Amelogenesis imperfecta: Diagnodis and resolution of a case with hypopiasia and hypocaicification of enamei, dentai agenesis, and skeietai open bite. UCLA Center for Esthetic Dentistry - Quintessence Int., 2001; 32 (3): 183-189.

17. Sari T., Usumez A. Restoring function and esthetics in a patient with amelogenesis imperfecta: a clinical report. J Prosthet Dent., 2003; 90(6): 522-525.

18. Nathwani NS, Kelleher M. Minimally destructive management of amelogenesis imperfecta and hypodontia with bleaching and bonding. Dent Update., 2010; 37 (3): 170-2, 175-6, 179.

19. Sadighpour L., Geramipanah F., Nikzad S. Fixed rehabilitation of an ACP PDI class III patient with amelogenesis imperfecta. J Prosthodont., 2009; 18(1): 64-70.

20. Peumans M., Van Meerbeek B., Lambrechts P., Vanharle G. Porcelain veneers: a review of the literature. J Dent., 2000; 28: 163-77.

21. Sholapurkar AA, Joseph RM, Varghese JM, Neelagiri K, Acharya SRR, Hedge V, et al. Clinical diagnosis and oral rehabilitation of a patient with amelogenesis imperfecta: a case report. J Contemp Dent Pract., 2008; 9(4): 1-11

22. Felippe LA, Baratieri LN, Monteiro JR S, Andrada MAC, Vieira LCC, Baroni R. Resinas condensáveis: como obter suas vantagens e evitar suas limitações. RGO., 2002; 50(3): 143-150.

\section{Dados dos autores}

\section{Rafaela Costa Holanda}

Mestranda em Odontopediatria pela Universidade Estadual de Campinas. Especialista em Odontopediatria pela Academia Cearense de Odontologia. Fortaleza/CE - Brasil. rafaela_ico@ hotmail.com

\section{Diana Maria Arraes Feitosa}

Graduação em Odontologia. pela Faculdade de Odontologia de Pernambuco. Especialista em Odontopediatria pela Associação Brasileira de Odontologia. Docente do curso de Especialização em Odontopediatria pela Academia Cearense de Odontologia. Fortaleza/CE - Brasil. clinicadianafeitosa@yahoo.com.br

\section{Ginna Kércia Matos Gonçalves \\ Mestre em Odontopediatria pela Universidade Cruzeiro do Sul. Coordenadora do curso de Especialização em Odontopediatria da Academia Cearense de Odontologia. Fortaleza/CE - Brasil. ginnagois@yahoo.com.br}

\section{Karla Shangela Silva Alves}

Doutora em Cariologia pela Universidade Federal do Ceará. Docente do curso de Odontologia do Centro Universitário Católica de Quixadá. Quixadá/ CE - Brasil. karla_shangela@hotmail.com

\section{Thayla Hellen Nunes Gouveia}

Mestre e Doutoranda em Dentística Restauradora pela Faculdade de Odontologia de Piracicaba Universidade Estadual de Campinas. Piracicaba/ SP - Brasil. thaylanunes@hotmail.com

\section{Patrícia Leal Dantas Lobo \\ Doutora em Farmacologia pela Universidade Federal do Ceará. Docente do curso de Odontologia da Universidade Federal do Ceará. Sobral/CE - Brasil.patricialdantas2@gmail.com}

Submetido em: 15-5-2017

Aceito em: 19-9-2017 